\title{
Trading Dynamics in Decentralized Markets with Adverse Selection
}

\author{
Braz Camargo \\ Benjamin Lester \\ University of Western Ontario \\ University of Western Ontario
}

February 12, 2010

\section{Preliminary and Incomplete}

\begin{abstract}
We study a dynamic, decentralized market environment with asymmetric information and interdependent values between buyers and sellers, and characterize the complete set of equilibria. The model delivers a stark relationship between the severity of the information frictions and the time it takes for the market to clear, or market liquidity. We use this framework to understand how asymmetric information has contributed to the "frozen" credit market at the core of the current financial crisis, and to characterize optimal policy responses to this market failure.
\end{abstract}




\section{Introduction}

A central problem in the current financial crisis has been the inability of financial institutions to sell illiquid assets on their balance sheets. More specifically, banks holding large amounts of structured asset-backed securities, such as collateralized debt obligations and credit default swaps, have been mostly unable to find buyers for these assets. This "frozen" market has posed perhaps the greatest risk to the economy as a whole; if financial institutions can not acquire liquid assets (e.g. cash) in exchange for these illiquid assets, they can not make loans. As a result, consumers have more difficulty buying cars and homes, and businesses cannot acquire the financing they need for new investment. This, in turn, can lead to a further decrease in asset prices and a decline in economic growth. Given the danger associated with this downward spiral, the task of identifying the underlying frictions in this market, and understanding the inefficiencies introduced by these frictions, is of crucial importance. Without such an understanding, market participants remain unsure of how this market will behave in the future, and policymakers remain unsure of the optimal form of intervention.

While one could point to a number of potential reasons that trade in this market has broken down, this paper focuses on asymmetric information. The story is simple: at the onset of the financial crisis, it became apparent that many assets being held by financial institutions were worth considerably less than had been previously claimed; they were of low quality or, in the language of Akerlof (1970), they were "lemons." Of course, these financial institutions also held assets of higher quality, whose fundamental value (though difficult to discern) was likely at or near pre-crisis evaluations. However, as these assets tend to be relatively complex, it was quite difficult to differentiate high quality from low. Thus the market had many of the basic ingredients of Akerlof's classic "market for lemons": sellers possessed assets that were heterogeneous in quality, and they were more informed about the quality of their assets than potential buyers. The most basic theory would predict that, in this type of environment, trade can break down completely.

However, there are several important features of this particular market that are not consistent with the assumptions typically embedded in existing models of markets with 
asymmetric information. For one, the market is decentralized; in contrast to the standard competitive paradigm, where the law of one price prevails, buyers and sellers in this market typically negotiate bilaterally. Therefore, a model of this market must allow the exchange of different quality assets to take place at potentially different prices. Moreover, the market is inherently dynamic and non-stationary; any serious analysis has to consider the manner in which the composition of assets in the market evolves over time, and how this affects both prices and the incentive of market participants to delay trade. In contrast to a static, centralized market environment, there are two mechanisms that can adjust to facilitate trade in a dynamic, decentralized market: prices and time.

In this paper, we develop a rigorous economic model that captures these important features of the market discussed above. To be more precise, we consider a discrete time, one-time entry model with a continuum of buyers and sellers. Sellers each possess a single good of heterogeneous quality, and this quality is private information. In each period, buyers and sellers are randomly matched, and buyers make a price offer chosen from an exogenously specified set of prices. If a seller accepts, trade ensues and the pair exits; if the seller rejects, the pair remain in the market and are randomly matched again the following period. Finally, we assume that agents are subject to stochastic discount factor shocks in each period, which we interpret as liquidity shocks across agents and over time. ${ }^{1}$

Within this environment, we address a variety of questions that are relevant in the current financial crisis. The first of these questions are positive: Will this market eventually clear and, if so, how long will it take? How does this length of time depend on the initial composition of high- and low-quality assets? What are the welfare costs associated with buyers being imperfectly informed? We will study these questions when the degree of informational asymmetry is exogenous, and when it is endogenous; i.e. when financial institutions can choose the quality of the assets they hold, and when potential investors can choose the degree to which they are uninformed about the quality of an asset before engaging in bilateral negotiations.

\footnotetext{
${ }^{1}$ This assumption also allows us to focus on pure strategies.
} 
Then we will turn to normative questions: Can government intervention increase welfare? If so, what is the optimal policy? This last point is particularly important in light of the variety of policy responses that have been either proposed or implemented since the financial crisis began. For example, one proposed policy has been for the federal government to buy assets directly from the sellers. An alternative, that has been implemented recently, is to essentially subsidize private-sector buyers to purchase assets from sellers. An important, open question that we intend to address is whether one of these policies implies larger efficiency gains than the other.

\section{$1.1 \quad$ Related Literature}

Our work builds on the literature that studies dynamic, decentralized markets with asymmetric information and interdependent values. The primary focus of this literature has been to determine what happens to equilibria in a decentralized environment as market frictions vanish. ${ }^{2}$ See Inderst (2005) and Moreno and Wooders (2009) for a steady-state analysis of this issue, and Moreno and Wooders (2002) and Blouin (2003) for analysis of this issue in a one-time entry model. Janssen and Roy (2002) also study a dynamic environment with asymmetric information and interdependent values; however, they assume is assumed takes place in a sequence of Walrasian markets. Though the framework we develop shares certain features in common with several of these papers, the focus will be quite different. We are interested in studying the relationship between information frictions and market liquidity (i.e. how long it takes for markets to clear), and the manner in which both market participants and policymakers can respond to overcome these frictions.

Our paper, and those discussed above, are also closely related to the literature that studies sequential bargaining between a single seller and a single buyer in the presence of asymmetric information. ${ }^{3}$ Most relevant to the current project is the work of Vincent (1989),

\footnotetext{
${ }^{2}$ Note that this was an exercise first conducted in a perfect information setting by Rubinstein and Wolinsky (1985) and Gale (1986a, 1986b). A parallel literature has emerged that studies dynamic, decentralized markets with imperfect information and private values; see, for example, Satterthwaite and Shneyerov (2007) and the references therein.

${ }^{3}$ Seminal contributions in this literature include Fudenberg et al. (1985) and Gul et al. (1986), among
} 
Evans (1989), and Deneckere and Liang (2006), who study the dynamic bargaining game in which a seller has private information about the quality of her good, a buyer makes offers in each period, and the buyer's valuation of the good is correlated with the seller's valuation. Equilibria in this environment tend to have the property that buyers use time to screen the different types of sellers: initially buyers will make low offers that only very low type sellers would accept. ${ }^{4}$ If the seller rejects such an offer, the buyer learns that the seller is not a very low type, and updates his posterior accordingly. In the following period, his offer increases, and so on.

This notion of using price dispersion over time to overcome the problem of adverse selection is central to our work, as well as the majority of papers cited above. ${ }^{5}$ What is different about the market setting we consider, as opposed to the single buyer/seller setting considered in much of the bargaining literature, is that complementarities can arise in a market setting between e.g. a buyer and other buyers. For example, in our setting multiple equilibria can arise, precisely because a single buyer will have greater incentive to delay trade if other buyers are doing the same. This type of complementarity between agents on the same side of the market is not present in an environment where there is only one agent on each side of the market; this is why there is generally a unique sequential equilibrium in the environments studied by Vincent (1989), Evans (1989), and Deneckere and Liang (2006).

\section{The Model}

Time is discrete, and begins in period $t=0$. There is an equal measure of infinitely lived buyers and sellers, which we normalize to one. Buyers are homogeneous, and discount the future at rate $\delta_{b}$. Sellers possess a single, indivisible asset. At $t=0$, a fraction $q_{0} \in(0,1)$ of these sellers possess a high quality $(H)$ asset, while the remaining sellers possess a low quality $(L)$ asset. An asset of quality $j \in\{L, H\}$ yields a buyer (seller) flow utility $y_{b}^{j}\left(y_{s}^{j}\right){ }^{6}$

others.

${ }^{4}$ A "low type seller" is a seller with a good of very low quality.

${ }^{5}$ This basic idea goes back to, at least, Wilson (1980).

${ }^{6}$ As in Duffie et al. (2005), our preference specification is such that buyers and sellers receive different levels of utility from holding a particular asset. This can arise for a multitude or reasons: agents can have 
The discount factor $\delta_{s}$ of a seller in each period is an i.i.d. draw from a continuous c.d.f. $F$ with support on $\left[0, \bar{\delta}_{s}\right]$, where $\bar{\delta}_{s}<1$. This is meant to capture the idea that sellers have different liquidity needs at different times. At a given time, some sellers may need to sell their asset urgently, while others may be more patient. Across time, each individual seller may be more or less patient in any given period. ${ }^{7}$

It will be convenient to denote the present discounted lifetime value of a type $j \in\{L, H\}$ asset to the buyer by $u_{j}$, where

$$
u_{j}=\frac{y_{b}^{j}}{1-\delta_{b}} .
$$

Similarly, we will denote the present expected discounted lifetime value of a type $j$ asset to a seller, computed before the seller draws his discount factor, by $c_{j}$, where

$$
c_{j}=\frac{y_{s}^{j}}{1-\mathbb{E}\left[\delta_{s}\right]},
$$

with $\mathbb{E}\left[\delta_{s}\right]=\int \delta_{s} d F\left(\delta_{s}\right)<1$. We normalize $y_{s}^{L}$ to zero, so that $c_{L}=0$ as well.

We assume that

$$
u_{H}>y_{s}^{H}+\bar{\delta}_{s} c_{H}>u_{L}>0
$$

The assumptions that $u_{H}>y_{s}^{H}+\bar{\delta}_{s} c_{H}$ and $u_{L}>0$ assure us that there are gains from trade in every match (i.e. from both low and high quality goods). The assumption that $y_{s}^{H}+\bar{\delta}_{s} c_{H}>u_{L}$ generates the lemons problem, as the price that buyers are willing to pay for a low quality asset would not be accepted by a sufficiently patient high quality seller.

In every period, after a seller draws a discount factor, buyers and sellers are randomly and anonymously matched in pairs. Both the seller's discount factor and the quality of his asset are private information. Once matched, the buyer can offer one of two prices: a high price

different levels of risk aversion, financing costs, regulatory requirements, or hedging needs. In addition, the correlation of endowments with asset returns may differ across agents. The current formulation is a reducedform representation of such differences; see Duffie et al. (2007), Vayanos and Weill (2008), and Gârleanu (2009).

${ }^{7}$ Note that both types of sellers draw their discount factors from the same distribution. Though this is non-essential, we think that it is reasonable. Moreover, we've assumed that buyers draw discount factors from a degenerate distribution. This, too, could be relaxed without altering the spirit of the results derived below. For a deeper look at the use of random discount factors, see Higashi et al. (2009). 
$p_{H} \in\left(y_{s}^{H}+\bar{\delta}_{s} c_{H}, u_{H}\right)$ or a low price $p_{L} \in\left(0, u_{L}\right) .{ }^{8}$ The seller can accept or reject. If a seller accepts, trade ensues and the pair exits the market; there is no entry by additional buyers and sellers. If a seller rejects, no trade occurs and the pair remains in the market. Note that this ensures that there is always an equal measure of buyers and sellers. We assume that

$$
u_{H}-p_{H}>u_{L}-p_{L}
$$

so that a buyer would choose to transact with a type $H$ seller if he could choose. We also assume that

$$
y_{s}^{H}+\bar{\delta}_{s} p_{H} \leq p_{H}
$$

As it turns out, (4) implies that a seller accepts an offer of $p_{H}$ regardless of his discount factor.

The history for a buyer is the set of all past (rejected) price offers. However, a buyer has no reason to condition behavior on his past history: this history is private information, and the probability that he meets his current trading partner in the future is zero, since there is a continuum of agents. Moreover, since there is no aggregate uncertainty in the model, the buyer's history of past offers is not helpful in learning any information about the aggregate state. Thus, a pure strategy for a buyer is a sequence $\mathbf{p}=\left\{p_{t}\right\}_{t=0}^{\infty}$ such that $p_{t}$ is the price he offers in period $t$ if he is in the market in this period.

A history for a seller is the set of all past discount factors and all price offers that he has rejected. Since discount factors are i.i.d., the same argument as above implies that a seller has no reason to condition behavior on his past history. Thus, a pure strategy for a seller with a type $j$ asset is a sequence $\mathbf{a}_{j}=\left\{a_{t}^{j}\right\}_{t=0}^{\infty}$, with $a_{t}^{j}:\left[0, \bar{\delta}_{s}\right] \times\left\{p_{L}, p_{H}\right\} \rightarrow\{$ accept, reject $\}$ measurable for all $t \geq 0$, such that $a_{t}^{j}\left(\delta_{s}, p\right)$ is the seller's acceptance decision in period $t$ as a function of his discount factor and the price offer he receives. We refer to $a_{t}^{j}$ as the seller's acceptance rule in period $t$.

We consider symmetric pure-strategy equilibria. A strategy profile can then be described by a list $\sigma=\left(\mathbf{p}, \mathbf{a}_{L}, \mathbf{a}_{H}\right)$. In order to define equilibria we need to specify what happens when

\footnotetext{
${ }^{8}$ Exogenous prices in these types of models have been used extensively; see, for example, Wolinsky (1990) and Blouin and Serrano (2001).
} 
there is a zero measure of agents remaining in the market; more specifically, when a mass one of agents trade and exit the market in the current period, we must specify the (expected) payoff to an individual should he choose a strategy that results in not trading. In order to avoid imposing ad hoc assumptions, we adopt the following procedure for computing payoffs.

Suppose that, in every period $t$, a fraction $\alpha \in(0,1]$ of buyers and sellers are matched in pairs, and the remainder do not get the opportunity to trade. The definition of strategies when $\alpha<1$ is the same as when $\alpha=1 .^{9}$ However, when $\alpha \in(0,1)$, in every period $t$ there is always a positive mass of agents who have not traded.

Let us denote by $V_{t}^{j}(\mathbf{a} \mid \sigma, \alpha)$ the expected payoff to a seller of type $j \in\{L, H\}$ who is in the market in period $t$ following strategy a, given the strategy profile $\sigma$ for all other agents. We assume that $V_{t}^{j}$ is computed before the seller gets the draw for his discount factor and learns whether he can trade or not. For $\alpha \in(0,1), V_{t}^{j}$ is well-defined for all $t \geq 0$, and satisfies the following recursion:

$$
\begin{aligned}
V_{t}^{j}(\mathbf{a} \mid \sigma, \alpha)= & (1-\alpha) \int\left[y_{s}^{j}+\delta_{s} V_{t+1}^{j}(\mathbf{a} \mid \sigma, \alpha)\right] d F\left(\delta_{s}\right) \\
& +\alpha \int\left\{\mathrm{I}_{t}^{j}\left(\delta_{s}, p_{t}\right) p_{t}+\left[1-\mathrm{I}_{t}^{j}\left(\delta_{s}, p_{t}\right)\right]\left[y_{s}^{j}+\delta_{s} V_{t+1}^{j}(\mathbf{a} \mid \sigma, \alpha)\right]\right\} d F\left(\delta_{s}\right),
\end{aligned}
$$

where $p_{t}$ is the price offered by the buyers in period $t$ and $\mathrm{I}_{t}^{j}\left(\delta_{s}, p_{t}\right)$ is the indicator function of the set $\left\{\delta_{s}: a_{t}\left(\delta_{s}, p_{t}\right)=\right.$ accept $\}$. In words, with probability $1-\alpha$ a seller is not matched in period $t$, enjoys flow utility $y_{s}^{j}$, and proceeds to period $t+1$. With probability $\alpha$ the seller is matched, in which case either the seller accepts the offer $p_{t}$ and exits the market, or rejects $p_{t}$ and stays in the market.

Similarly, we denote by $V_{t}^{b}(\mathbf{p} \mid \sigma, \alpha)$ the expected payoff to a buyer who is in the market in period $t$ following strategy $\mathbf{p}$, given the strategy profile $\sigma$ for all other agents. Again, for

\footnotetext{
${ }^{9}$ Now a history for a player also includes the periods in which he was able to trade; for the same reasons given above, a player has no motive to condition his behavior on this information.
} 
$\alpha \in(0,1), V_{t}^{b}$ is well-defined for all $t \geq 0$, and satisfies the following recursion:

$$
\begin{aligned}
V_{t}^{b}(\mathbf{p} \mid \sigma, \alpha)=(1-\alpha) \delta_{b} V_{t+1}^{b}(\mathbf{p} \mid \sigma, \alpha)+\alpha & \left\{q_{t} \lambda_{t}^{H}\left(p_{t}\right)\left[u_{H}-p_{t}\right]+\left(1-q_{t}\right) \lambda_{t}^{L}\left(p_{t}\right)\left[u_{L}-p_{t}\right]\right. \\
+ & \left.+\left[1-q_{t} \lambda_{t}^{H}\left(p_{t}\right)-\left(1-q_{t}\right) \lambda_{t}^{L}\left(p_{t}\right)\right] \delta_{b} V_{t+1}^{b}(\mathbf{p} \mid \sigma, \alpha)\right\} \\
= & \delta_{b} V_{t+1}^{b}(\mathbf{p} \mid \sigma, \alpha)+\alpha\{ \\
& q_{t} \lambda_{t}^{H}\left(p_{t}\right)\left[u_{H}-p_{t}-\delta_{b} V_{t+1}^{b}(\mathbf{p} \mid \sigma, \alpha)\right] \\
& \left.+\left(1-q_{t}\right) \lambda_{t}^{L}\left(p_{t}\right)\left[u_{L}-p_{t}-\delta_{b} V_{t+1}^{b}(\mathbf{p} \mid \sigma, \alpha)\right]\right\}
\end{aligned}
$$

where $\lambda_{t}^{j}\left(p_{t}\right)$ is the likelihood that a seller of type $j \in\{L, H\}$ in the market in period $t$ accepts an offer $p_{t} \in\left\{p_{L}, p_{H}\right\}$ and $q_{t}$ is the fraction of $H$ sellers in the market in period $t$.

Standard dynamic programming arguments show that for each $\sigma, \mathbf{a}, \mathbf{p}$, and $t \geq 0$, the payoffs $V_{t}^{j}(\mathbf{a} \mid \sigma, \alpha)$ and $V_{t}^{b}(\mathbf{p} \mid \sigma, \alpha)$ are continuous functions of $\alpha$ in the interval $(0,1)$. Hence, the limits of both $V_{t}^{j}(\mathbf{a} \mid \sigma, \alpha)$ and $V_{t}^{b}(\mathbf{p} \mid \sigma, \alpha)$ are well-defined as $\alpha$ converges to one.

Definition 1. Let $\sigma$ be the strategy profile under play. The payoff to a buyer who is in the market in period $t$ and follows the strategy $\mathbf{p}$ from $t$ on is $V_{t}^{b}(\mathbf{p} \mid \sigma)=\lim _{\alpha \rightarrow 1} V_{t}^{b}(\mathbf{p} \mid \sigma, \alpha)$. The payoff to a seller of type $j \in\{L, H\}$ who is in the market in period $t$ and follows the strategy a from $t$ on is $V_{t}^{j}(\mathbf{a} \mid \sigma)=\lim _{\alpha \rightarrow 1} V_{t}^{j}(\mathbf{a} \mid \sigma, \alpha)$.

We can now define equilibria in our environment.

Definition 2. The strategy profile $\sigma^{*}=\left(\mathbf{p}^{*}=\left\{p_{t}^{*}\right\}, \mathbf{a}_{L}^{*}=\left\{a_{t}^{L *}\right\}, \mathbf{a}_{H}^{*}=\left\{a_{t}^{H *}\right\}\right)$ is an equilibrium if for each $t \geq 0$ and $j \in\{L, H\}$, we have that:

(i) $p_{t}^{*}$ maximizes

$$
q_{t} \lambda_{t}^{H}(p)\left[u_{H}-p-\delta_{b} V_{t+1}^{b}\left(\sigma^{*}\right)\right]+\left(1-q_{t}\right) \lambda_{t}^{L}(p)\left[u_{L}-p-\delta_{b} V_{t+1}^{b}\left(\sigma^{*}\right)\right],
$$

where $V_{t}^{b}\left(\sigma^{*}\right)=V_{t}^{b}\left(\mathbf{p}^{*} \mid \sigma^{*}\right)$;

(ii) For each $p \in\left\{p_{L}, p_{H}\right\}, a_{t}^{j *}\left(\delta_{s}, p\right)=$ accept if, and only if,

$$
p \geq y_{s}^{j}+\delta_{s} V_{t+1}^{j}\left(\sigma^{*}\right)
$$

where $V_{t}^{j}\left(\sigma^{*}\right)=V_{t}^{j}\left(\mathbf{a}_{j}^{*} \mid \sigma^{*}\right)$. 
Note that (7) implies that in equilibrium a seller accepts any offer that he is indifferent between accepting and rejecting. This is without loss since $F_{s}$ is continuous, and so the probability that a seller is ever indifferent between accepting and rejecting is zero.

\section{Preliminary Results}

For a given strategy profile, we say that the market "clears" in period $k$ if all sellers remaining in the market accept the price offer made by the buyers. In this section we establish two preliminary results. First, that an equilibrium exists regardless of the initial fraction $q_{0}$ of $H$ sellers. Second, that the market clears in finite time in every equilibrium.

Proposition 1. An equilibrium exists for all $q_{0} \in(0,1)$.

\section{Proof. TO BE ADDED.}

In order to establish the second result of this section, we first show that the market clears in period $t$ only if $p_{t}=p_{H}$. From (5) and (7), we have that for any equilibrium strategy $\sigma^{*}$,

$$
V_{t}^{j}\left(\sigma^{*}\right)=\int \max \left\{p_{t}, y_{s}^{j}+\delta_{s} V_{t+1}^{j}\left(\sigma^{*}\right)\right\} d F\left(\delta_{s}\right)
$$

for all $t \geq 0$. Given (4), it should be obvious that $V_{t}^{j}\left(\sigma^{*}\right) \leq p_{H}$ for all $t \geq 0$, so that all sellers in the market always accept an offer of $p_{H}$.

Moreover, so long as $q_{0}>0$, a strictly positive measure of agents will always reject $p_{L}$. To see this, first note that $q_{t}$ is weakly increasing in any equilibrium (we show this below), so that $q_{0}>0$ implies that $q_{t}>0$ for all $t>0$ in which there remain a strictly positive mass of agents in the market. Therefore, consider any period $t$ with $q_{t}>0$ and $p_{t}=p_{L}$.

Since a seller has the option of always rejecting any offer he receives, $V_{t}^{j}\left(\sigma^{*}\right) \geq c_{j}$ for every period $t$. Thus, letting $\underline{\delta}_{s}=\left(u_{L}-y_{s}^{H}\right) / c_{H}$, by construction we have

$$
y_{s}^{H}+\underline{\delta}_{s} V_{t+1}^{H}\left(\sigma^{*}\right) \geq y_{s}^{H}+\underline{\delta}_{s} c_{H}=u_{L}>p_{L} .
$$

Therefore, a type $H$ seller with discount factor $\delta_{s} \geq \underline{\delta}_{s}$ always rejects an offer of $p_{L}$. Since $\underline{\delta}_{s}<\bar{\delta}_{s}$ by (3), there is always a strictly positive mass of such buyers. 
Given this result, we conclude that the market clears in the first period in which the buyers offer $p_{H}$, which we denote by $T=T\left(\sigma^{*}\right) \cdot{ }^{10}$ For all $t<T$, buyers offer $p_{t}=p_{L}$, and the fraction of type $j$ sellers who accept $p_{L}$ in $t$ is $F\left[\left(p_{L}-y_{s}^{j}\right) / V_{t+1}^{j}\left(\sigma^{*}\right)\right]$. Thus, for all $t \in\{1, \ldots, T-1\}$

$$
q_{t+1}=\frac{q_{t}\left[1-F\left(\frac{p_{L}-y_{s}^{H}}{V_{t+1}^{H}\left(\sigma^{*}\right)}\right)\right]}{q_{t}\left[1-F\left(\frac{p_{L}-y_{s}^{H}}{V_{t+1}^{H}\left(\sigma^{*}\right)}\right)\right]+\left(1-q_{t}\right)\left[1-F\left(\frac{p_{L}}{V_{t+1}^{L}\left(\sigma^{*}\right)}\right)\right]} .
$$

Now notice that an option for a type $H$ seller is to replicate the behavior of a type $L$ seller. Since $y_{s}^{H}>y_{s}^{L}$, we then have that $V_{t}^{H}\left(\sigma^{*}\right) \geq V_{t}^{L}\left(\sigma^{*}\right)$ for all $t \geq 0 .{ }^{11}$ Hence, since $F\left(\left(p_{L}-y_{s}^{H}\right) / V_{t+1}^{H}\left(\sigma^{*}\right)\right) \leq F\left(\underline{\delta}_{s}\right)<1$,

$$
F\left(\frac{p_{L}-y_{s}^{H}}{V_{t+1}^{H}\left(\sigma^{*}\right)}\right)<F\left(\frac{p_{L}}{V_{t+1}^{L}\left(\sigma^{*}\right)}\right)
$$

for all $t \geq 0$; that is, whenever buyers offer $p_{L}$, the fraction of type $L$ sellers who accept is larger than the fraction of type $H$ sellers who accept. This is a fundamental feature of this environment: type $H$ sellers are de facto more patient than type $L$ sellers because their flow payoff from not trading is larger. Looking at the law of motion for $\left\{q_{t}\right\}$, equation (8), a consequence of this fact is that the fraction of type $H$ sellers in the population increases strictly over time before the market clears.

Proposition 2. Let $q_{0} \in(0,1)$. In any equilibrium, the market clears in finite time.

Proof. Let $\sigma^{*}$ be an equilibrium and assume, by contradiction, that $T=\infty$. This implies that $V_{t}^{L}\left(\sigma^{*}\right)=p_{L}$ for all $\geq 0$ : if a type $L$ seller in the market expects the buyers to offer $p_{L}$ in every period, then it is optimal for him to accept an offer of $p_{L}$ immediately. In particular, $F\left[p_{L} / V_{1}^{L}\left(\sigma^{*}\right)\right]=1$, and so $q_{1}=1$. However, it is uniquely optimal for a buyer to offer $p_{H}$

\footnotetext{
${ }^{10}$ We set $T=\infty$ if the buyer offers $p_{L}$ in every period, though we establish below that this is never part of an equilibrium.

${ }^{11}$ Indeed, $y_{s}^{H}>y_{s}^{L}$ implies that $V_{t}^{H}\left(\mathbf{a}_{L} \mid \sigma^{*}, \alpha\right)>V_{t}^{L}\left(\mathbf{a}_{L} \mid \sigma^{*}, \alpha\right)$ for all $\alpha \in(0,1)$ and $t \geq 0$. Thus, $V_{t}^{H}\left(\sigma^{*}\right)=\lim _{\alpha \rightarrow 1} V_{t}^{H}\left(\mathbf{a}_{H} \mid \sigma^{*}, \alpha\right) \geq \lim _{\alpha \rightarrow 1} V_{t}^{H}\left(\mathbf{a}_{L} \mid \sigma^{*}, \alpha\right) \geq \lim _{\alpha \rightarrow 1} V_{t}^{L}\left(\mathbf{a}_{L} \mid \sigma^{*}, \alpha\right)=V_{t}^{L}\left(\sigma^{*}\right)$.
} 
when the fraction of type $H$ sellers in the market is one. Thus, the market clears in $t=1$, a contradiction.

In what follows, we will present the case where the lemons problems is most severe by assuming that $p_{L}<y_{s}^{H}$, so that no type $H$ seller accepts $p_{L}$. Relaxing this assumption does not substantively change any of our results. We also assume that

$$
\delta_{b} \leq\left(u_{L}-p_{L}\right) /\left(u_{H}-p_{H}\right)
$$

which ensures that the buyer would never prefer to simply not make an offer.

\section{Characterizing Equilibria}

In this section we provide a complete characterization of the set of equilibria. We start with a characterization of the equilibria in which the market clears immediately. We refer to such equilibria as 0 -step equilibria. In general, we refer to equilibria in which the market clears in period $k$, with $k \geq 0$, as $k$-step equilibria.

\section{0-step equilibria.}

Denote by $\pi_{b}^{k}\left(q, v_{L}, v_{H}, v_{b}\right)$ the payoff to a buyer who offers $p_{k}$ for $k \in\{L, H\}$ when: $(i)$ the fraction of type $H$ sellers in the market is $q$; $(i i)$ the continuation payoff to a seller of type $j$ who chooses not trade is $v_{j}$; and $(i i i)$ the continuation payoff to the buyer should he not trade is $v_{b}$. We know that sellers always accept an offer of $p_{H}$. So,

$$
\pi_{b}^{H}\left(q, v_{L}, v_{H}, v_{b}\right)=\pi_{b}^{H}(q)=q\left[u_{H}-p_{H}\right]+(1-q)\left[u_{L}-p_{H}\right]
$$

We also know that a type $H$ seller always rejects an offer of $p_{L}$. So,

$$
\begin{gathered}
\pi_{b}^{L}\left(q, v_{L}, v_{H}, v_{b}\right)=\pi_{b}^{L}\left(q, v_{L}, v_{b}\right)= \\
(1-q) F\left(\frac{p_{L}}{v_{L}}\right)\left[u_{L}-p_{L}\right]+\left\{q+(1-q)\left[1-F\left(\frac{p_{L}}{v_{L}}\right)\right]\right\} \delta_{b} v_{b},
\end{gathered}
$$

where $F\left(p_{L} / v_{L}\right)$ is the fraction of type $L$ sellers who accept $p_{L}$. Notice that $\pi_{b}^{L}\left(q, v_{L}, v_{b}\right)$ is increasing in $v_{b}$. Since $v_{b} \leq u_{H}-p_{H}$, (9) implies that $\pi_{b}^{L}\left(q, v_{L}, v_{b}\right)$ is decreasing in $v_{L}$. 
Consider now the candidate 0 -step equilibrium profile $\sigma^{0}$ where the buyers always offer $p_{H}$, and in every period $t$ the type $j$ sellers accept an offer $p$ if, and only if, $\delta_{s} \leq\left(p-y_{s}^{j}\right) / p_{H}{ }^{12}$ It is immediate to see that for all $t \geq 0$,

$$
V_{t}^{b}\left(\sigma^{0}\right) \equiv v_{b}^{0}\left(q_{0}\right)=q_{0}\left[u_{H}-p_{H}\right]+\left(1-q_{0}\right)\left[u_{L}-p_{H}\right],
$$

and $V_{t}^{j}\left(\sigma^{0}\right)=v_{0}^{j}=p_{H}$ for $j \in\{L, H\}$. Note that we have introduced the following notation: in a 0 -step equilibrium, the expected payoff to a buyer given $q_{0}$ is $v_{b}^{0}\left(q_{0}\right)$ and the expected payoff to a type $j$ seller is $v_{j}^{0}$.

The strategy profile $\sigma^{0}$ is an equilibrium as long as in every period $t$ the buyers find it optimal to offer $p_{H}$, which is true as long as

$$
\pi_{b}^{H}(q) \geq \pi_{L}^{b}\left(q, v_{L}^{0}, v_{b}^{0}(q)\right)
$$

This condition is slightly more subtle than it may appear. The left side of the inequality above is clearly the payoff to an individual buyer from offering $p_{H}$. The right hand side is the payoff to an individual buyer from offering $p_{L}$ in the current period and $p_{H}$ in the ensuing period, conditional on all other buyers offering $p_{H}$ in the current period. There are two things to notice. First, that when all other buyers offer $p_{H}$, the fraction of $H$ sellers in the market does not change between this period and next; this comes directly from our refinement of considering payoffs for $\alpha \in(0,1)$ as $\alpha$ converges to 1 . Second, as we show more formally in the proof of Proposition 3 below, by assuming that the individual buyer offers $p_{H}$ in the ensuing period, (11) is the loosest possible constraint on $q$.

Proposition 3. Let $\underline{q}^{0} \in(0,1)$ denote the unique value of $q$ satisfying

$$
\pi_{b}^{L}\left(q, v_{L}^{0}, v_{b}^{0}(q)\right)=\pi_{b}^{H}(q)
$$

There exists a 0-step equilibrium if, and only if, $q_{0} \geq \underline{q}^{0}$.

\footnotetext{
${ }^{12}$ In general, we will adopt the convention that a numerical subscript refers to a particular time period $(t=0,1,2, \ldots)$, while a numerical superscript refers to the number of periods it takes for the market to clear in equilibrium $(k=0,1,2, \ldots)$.
} 
Proof. First, let $\eta^{0}(q)=\pi_{b}^{H}(q)-\pi_{b}^{L}\left(q, v_{L}^{0}, v_{b}^{0}(q)\right)$. Straightforward algebra shows that $\eta^{0}$ is strictly increasing. Since $\eta^{0}$ is continuous, $\eta^{0}(0)<0$, and $\eta^{0}(1)>0$, we can conclude that there is a unique value of $q$ in $(0,1)$, that we denote by $\underline{q}^{0}$, such that $\eta^{0}\left(\underline{q}^{0}\right) \geq 0$ if, and only if $q \geq \underline{q}^{0}$. In other words, $\pi_{b}^{H}(q) \geq \pi_{b}^{L}\left(q, v_{L}^{0}, v_{b}^{0}(q)\right)$ if, and only if, $q \geq \underline{q}^{0}$. This assures no profitable one-shot deviations, and thus the existence of a 0-step equilibrium for any $q_{0} \geq \underline{q}^{0}$.

Now suppose $q_{0}<\underline{q}^{0}$, and consider a candidate 0 -step equilibrium profile $\hat{\sigma}$ with the necessary property that $p_{0}=p_{H}$. Notice that one alternative for a buyer is to offer $p_{H}$ in every period. Let $\widetilde{p}_{H}$ denote this strategy. If $\hat{\sigma}$ is to be an equilibrium, then it must be that $V_{t}^{b}(\hat{\sigma}) \geq V_{t}^{b}\left(\widetilde{p}_{H} \mid \hat{\sigma}\right)$ for all $t \geq 0$. Moreover, we know that as long as there is a positive mass of agents in the market, the fraction of type $H$ sellers does not decrease over time. From this fact one can show that $V_{t}^{b}\left(\widetilde{p}_{H} \mid \hat{\sigma}\right) \geq v_{b}^{0}\left(q_{0}\right)$ for all $t \geq 0$; the details are in the Appendix. Therefore, since $\pi_{b}^{L}\left(q, v_{L}^{0}, v_{b}^{0}(q)\right)>\pi_{b}^{H}(q)$ for $q_{0}<\underline{q}^{0}$, we have

$$
\pi_{b}^{L}\left(q, V_{1}^{L}(\hat{\sigma}), V_{1}^{b}(\hat{\sigma})\right) \geq \pi_{b}^{L}\left(q, v_{L}^{0}, v_{b}^{0}(q)\right)>\pi_{b}^{H}(q) .
$$

Thus, it can not be optimal for a buyer to offer $p_{H}$ at $t=0$ if $q_{0}<\underline{q}^{0}$, so that the market clearing immediately cannot be an equilibrium outcome.

Corollary 1. Consider two distributions $F\left(\delta_{s}\right)$ and $\hat{F}\left(\delta_{s}\right)$ with associated cutoffs $\underline{q}^{0}$ and $\underline{\hat{q}}^{0}$, respectively. If $\hat{F}$ dominates $F$ in the first order stochastic sense, then $\underline{q}^{0}>\underline{\hat{q}}^{0}$.

Proof. Note that $\underline{q}^{0}$ satisfies

$$
(1-q) F\left(\frac{p_{L}}{v_{L}^{0}}\right)\left[u_{L}-p_{L}-\delta_{b} v_{b}^{0}(q)\right]-\left(1-\delta_{b}\right) v_{b}^{0}(q)=0,
$$

where $v_{L}^{0}=p_{H}$. Straight-forward algebra shows an increase in $F\left(\frac{p_{L}}{v_{L}^{0}}\right)$ leads to an increase in $\underline{q}^{0}$.

In words, corollary 1 implies that as seller become more impatient, the cutoff $\underline{q}^{0}$ increases and thus the set of 0-step equilibria shrinks. The reason is simple: as sellers become more impatient, buyers have greater incentive to offer $p_{0}=p_{L}$, since it becomes more likely to be accepted, as opposed to $p_{0}=p_{H}$ which would clear the market. The highest value of $\underline{q}^{0}$ is 
achieved when $\bar{\delta}_{s} \leq p_{L} / v_{L}^{0}$, in which case $\underline{q}^{0}$ is the unique solution to $u_{L}-p_{L}=\delta_{b} v_{b}^{0}(q)$. It is easy to see that $\underline{q}^{0}$ is never smaller than the value of $q$ for which $v_{b}^{0}(q)=0$.

Notice that $q_{0} u_{H}+\left(1-q_{0}\right) u_{L} \geq p_{H}>y_{s}^{H}+\bar{\delta}_{s} c_{H}$ for any $q_{0}$ in the interval $\left[\underline{q}^{0}, 1\right)$, since a buyer is only willing to offer $p_{H}$ if his payoff from doing so is non-negative. ${ }^{13}$ Hence, $p_{H}$ corresponds to a market clearing price in a Walrasian equilibrium. Thus, when the lemons problem is relatively small, i.e., when the fraction of type $H$ sellers is sufficiently large, the market behaves as if it were Walrasian. We will now show that when the lemons problem gets severe, the market outcomes no longer resemble those of a centralized Walrasian market; instead, these markets appear more like standard decentralized search markets, in the sense that it takes time for buyers and sellers to trade, and they do so at potentially different prices.

\section{1-step equilibria.}

As the next step, we characterize the set of 1-step equilibria. For this, let

$$
q^{+}\left(q, v_{L}\right)=\frac{q}{q+(1-q)\left[1-F\left(\frac{p_{L}}{v_{L}}\right)\right]}
$$

By construction, $q^{+}\left(q, v_{L}\right)$ is the fraction of type $H$ sellers in the market in the next period if in the current period this fraction is $q$, the buyers offer $p_{L}$, and the continuation payoff to a type $L$ seller in case he rejects a price offer is $v_{L}$. Notice that if $p_{L} / v_{L}<\bar{\delta}_{s}$, then $q^{+}\left(q, v_{L}\right)$ is strictly increasing in $q$, and that if $p_{L} / v_{L} \geq \bar{\delta}_{s}$, then $q^{+}\left(q, v_{L}\right) \equiv 1$.

Consider a strategy profile $\sigma^{1}=\left\{\sigma_{t}^{1}\right\}_{t=0}^{\infty}$ such that the buyers offer $p_{L}$ in $t=0$ and $p_{H}$ in $t=1$. In order for $\sigma^{1}$ to be an equilibrium, it must be that $\sigma^{1+}=\left\{\sigma_{t}\right\}_{t=1}^{\infty}$ is a 0 -step equilibrium at $t=1$ when the initial fraction of type $H$ sellers is $q^{+}\left(q_{0}, v_{L}^{0}\right) .{ }^{14}$ Hence, the

\footnotetext{
${ }^{13}$ This is true because a buyer has the option of always offering $p_{L}$ for a non-negative payoff.

${ }^{14}$ It must also be the case that the type $j$ sellers accept an offer of $p$ in $t=0$ if, and only if, $\delta_{s} \leq\left(p-y_{s}^{j}\right) / p_{H}$. This optimal behavior of sellers will often be implicitly assumed throughout the analysis.
} 
following two conditions are necessary and sufficient condition for $\sigma^{1}$ to be an equilibrium:

$$
\begin{gathered}
q^{+}\left(q_{0}, v_{L}^{0}\right) \geq \underline{q}^{0} \\
\pi_{b}^{H}\left(q_{0}\right) \leq \pi_{b}^{L}\left(q_{0}, v_{L}^{0}, v_{b}^{0}\left[q^{+}\left(q_{0}, v_{L}^{0}\right)\right]\right) .
\end{gathered}
$$

Condition (12) follows from Proposition 3. It ensures that the fraction of type $H$ sellers in $t=1$ is high enough for market clearing in this period to be an equilibrium outcome. Condition (13) ensures that it is optimal for a buyer to offer $p_{L}$ in $t=0$ when all other buyers offer $p_{L}$ as well and the market clears in $t=1$.

In a 1-step equilibrium, the payoff to a buyer is

$$
v_{b}^{1}(q)=\pi_{b}^{L}\left(q, v_{L}^{0}, v_{b}^{0}\left[q^{+}\left(q, v_{L}^{0}\right)\right]\right)
$$

and the payoff to a type $L$ seller is

$$
v_{L}^{1}=\int \max \left\{p_{L}, \delta_{s} v_{L}^{0}\right\} d F\left(\delta_{s}\right)<v_{L}^{0} .
$$

If $\bar{\delta}_{s}>p_{L} / v_{L}^{0}$, we can use integration by parts to show that

$$
v_{L}^{1}=p_{H}\left\{\bar{\delta}_{s}-\int_{p_{L} / v_{L}^{0}}^{\bar{\delta}_{s}} F\left(\delta_{s}\right) d \delta_{s}\right\}
$$

Proposition 4. There exist $0 \leq \underline{q}^{1}<\underline{q}^{0}<\bar{q}^{1}<1$ such that a 1 -step equilibrium exists if, and only if, $q_{0} \in\left[\underline{q}^{1}, \bar{q}^{1}\right]$. The upper cutoff $\bar{q}^{1}$ is the unique value of $q$ for which

$$
\pi_{b}^{H}(q)=\pi_{b}^{L}\left(q, v_{L}^{0}, v_{b}^{0}\left[q^{+}\left(q, v_{L}^{0}\right)\right]\right)
$$

If $p_{L} / v_{L}^{0} \geq \bar{\delta}_{s}$, then $\underline{q}^{1}=0$. Otherwise, $\underline{q}^{1}$ is the unique value of $q$ such that

$$
q^{+}\left(q, v_{L}^{0}\right)=\underline{q}^{0} .
$$

In words, if $q_{0}=\bar{q}^{1}$, then a buyer is exactly indifferent between offering $p_{L}$ and $p_{H}$ when all other buyers are offering $p_{L}$; for any $q_{0}>\bar{q}^{1}$ the payoff from immediately trading at price $p_{H}$ is greater than the payoff from trading with some probability at price $p_{L}$ in the current period, and trading with some probability at price $p_{H}$ in the ensuing period (albeit with a 
greater probability of purchasing a high quality good). If even the most patient type $L$ seller would rather accept an offer of $p_{L}$ today than wait one period for an offer of $p_{H}$, then we have $\underline{q}_{1}=0$. Otherwise, $\underline{q}^{1}$ is such that if $q_{0}=\underline{q}^{1}$ and all buyers offer $p_{L}$, then the fraction of high quality sellers in the next period will be exactly $\underline{q}^{0}$, the minimum value of the fraction of type $H$ sellers needed for market clearing.

Proof of Proposition 4. Since $q^{+}\left(q, v_{L}^{0}\right)$ is strictly increasing in $q$ when $p_{L} / v_{L}^{0}<\bar{\delta}_{s}$ and $q^{+}\left(q, v_{L}^{0}\right) \equiv 1$ otherwise, it is immediate to see that there is $\underline{q}^{1}<\underline{q}^{0}$ such that (12) is satisfied if, and only if, $q_{0} \in\left[\underline{q}^{1}, 1\right]$. Notice that $\underline{q}^{1}=0$ if $p_{L} / v_{L}^{0} \geq \bar{\delta}_{s}$ and $q^{+}\left(\underline{q}^{1}, v_{L}^{0}\right)=\underline{q}^{0}$ otherwise. Now let

$$
\eta^{1}(q)=\pi_{b}^{H}(q)-\pi_{b}^{L}\left(q, v_{L}^{0}, v_{b}^{0}\left[q^{+}\left(q, v_{L}^{0}\right)\right]\right)
$$

Straightforward algebra shows that

$$
\begin{aligned}
\frac{\partial \eta^{1}}{\partial q}=F\left(\frac{p_{L}}{p_{H}}\right)\left\{u_{L}\right. & \left.-p_{L}-\delta_{b} v_{b}^{0}\left[q^{+}\left(q, p_{H}\right)\right]\right\} \\
& +\left(u_{H}-u_{L}\right)\left\{1-\delta_{b}\left\{q+(1-q)\left[1-F\left(\frac{p_{L}}{p_{H}}\right)\right]\right\} \frac{\partial q^{+}}{\partial q}\right\},
\end{aligned}
$$

from which we can conclude, by (9), that $\partial \eta^{1} / \partial q>0$ regardless of the value of $p_{L} / p_{H}$. Since $\eta^{1}(0)<0$ and $\eta^{1}(1)>0$, we then have that there exits $\bar{q}^{1} \in(0,1)$ such that (13) is satisfied if, and only if $q_{0} \in\left[0, \bar{q}^{1}\right]$. To finish, observe that since $v_{b}^{0}\left[q^{+}\left(q, p_{H}\right)\right]>v_{b}^{0}(q)$ for all $q \in(0,1)$,

$$
\pi_{b}^{L}\left(q, p_{H}, v_{b}^{0}\left[q^{+}\left(\underline{q}^{0}, p_{H}\right)\right]\right)>\pi_{b}^{L}\left(q, p_{H}, v_{b}^{0}\left(\underline{q}^{0}\right)\right)=\pi_{b}^{H}\left(\underline{q}^{0}\right)
$$

and so $\bar{q}^{1}>q^{0}$.

Notice that $\underline{q}^{0}<\bar{q}^{1}$ implies that there are multiple equilibria when $q_{0} \in\left[\underline{q}^{0}, \bar{q}^{1}\right]$. In this region, if all other buyers are offering $p_{H}$, it is optimal for an individual buyer to offer $p_{H}$. However, if all other buyers are offering $p_{L}$, the market does not clear at $t=0$ and the payoff to trading at $t=1$ increases, rendering it optimal for buyers to offer $p_{0}=p_{L}$ and incur a chance that they trade only in the next period. 


\section{$k$-step equilibria}

We are now in position to provide a complete characterization of the $k$-step equilibria for all $k \geq 2$. Recall from Proposition 4 that if $p_{L} / p_{H} \geq \bar{\delta}_{s}$, then the only equilibria possible are 0 -step and 1-step equilibria. Given this, we assume that $p_{L} / p_{H}<\bar{\delta}_{s}$ in what follows. As a first step we, characterize the 2-step equilibria.

Consider a strategy profile $\sigma^{2}=\left\{\sigma_{t}^{2}\right\}_{t=0}^{\infty}$ such that the buyers offer $p_{L}$ in the first two periods, and then offer $p_{H}$. In order for $\sigma^{2}$ to be an equilibrium, it must be that $\sigma^{2+}=\left\{\sigma_{t}^{2}\right\}_{t=1}^{\infty}$ is a 1 -step equilibrium when the fraction of type $H$ sellers is $q^{+}\left(q_{0}, v_{L}^{1}\right)$. Hence, the following two conditions are necessary and sufficient for $\sigma^{2}$ to be an equilibrium:

$$
\begin{gathered}
q^{+}\left(q_{0}, v_{L}^{1}\right) \in\left[\underline{q}^{1}, \bar{q}^{1}\right] \\
\pi_{b}^{H}\left(q_{0}\right) \leq \pi_{b}^{L}\left(q_{0}, v_{L}^{1}, v_{b}^{1}\left[q^{+}\left(q_{0}, v_{L}^{1}\right)\right]\right) .
\end{gathered}
$$

Condition (15) follows from Proposition 4. Condition (16) ensures that it is optimal for the buyers to offer $p_{L}$ in $t=0$ when all other buyers do the same and the behavior from $t=1$ on is that of a 1-step equilibrium.

First notice that a necessary condition for (15) to hold is that

$$
\frac{p_{L}}{v_{L}^{1}}<\bar{\delta}_{s} .
$$

Condition (17) ensures that if the continuation payoff to the type $L$ sellers who do not transact is $v_{L}^{1}$, then a positive mass of these sellers rejects an offer of $p_{L}$ in $t=0$. Otherwise, the fraction of type $H$ sellers in the population increases to 1 in the second period, which is not compatible with a 2-step equilibrium. It is possible to show that for each value of $\bar{\delta}_{s}$ there is an upper bound on $p_{L} / p_{H}$ below which (17) is satisfied. Moreover, one can show that this upper bound increases to 1 as $\bar{\delta}_{s}$ increases to 1 . In what follows we assume that (17) is satisfied.

We now show that all values of $q_{0}$ that satisfy (15) also satisfy (16), so that condition (15) completely characterizes the range of initial values of $q_{0}$ for which there exists a 2-step 
equilibrium. Before we start, notice that

$$
\left\{q+(1-q)\left[1-F\left(\frac{p_{L}}{v_{L}}\right)\right]\right\} q^{+}\left(q, v_{L}\right)=q
$$

for all $q \in(0,1)$ and $v_{L} \geq p_{L}$. Hence,

$$
\pi_{b}^{L}\left(q, v_{L}, v_{b}^{0}\left[q^{+}\left(q, v_{L}\right)\right]\right)=\delta_{b} v_{b}^{0}(q)+(1-q) F\left(\frac{p_{L}}{v_{L}}\right)\left[u_{L}-p_{L}-\delta_{b}\left(u_{L}-p_{H}\right)\right] .
$$

Equation (18) is useful in what follows.

Let $q_{0}$ be such that $q^{\prime}=q^{+}\left(q_{0}, v_{L}^{1}\right) \in\left[q^{1}, \bar{q}^{1}\right]$. Notice that $(17)$ implies that $q^{+}\left(q_{0}, v_{L}^{1}\right)$ is greater then $q_{0}$. By construction,

$$
v_{b}^{1}\left(q^{\prime}\right)=\pi_{b}^{L}\left(q^{\prime}, v_{L}^{0}, v_{b}^{0}\left[q^{+}\left(q^{\prime}, v_{L}^{0}\right)\right]\right) \geq \pi_{b}^{H}\left(q^{\prime}\right)=v_{b}^{0}\left(q^{\prime}\right)
$$

Given (19), in order to prove that (16) is satisfied, it is sufficient to show

$$
\pi_{b}^{H}\left(q^{\prime}\right)-\pi_{b}^{H}\left(q_{0}\right) \geq \pi_{b}^{L}\left(q^{\prime}, v_{L}^{0}, v_{b}^{0}\left[q^{+}\left(q^{\prime}, v_{L}^{0}\right)\right]\right)-\pi_{b}^{L}\left(q_{0}, v_{L}^{1}, v_{b}^{1}\left(q^{\prime}\right)\right)
$$

Condition (20) implies that a buyer's incentive to choose $p_{L}$ in $t=0$ is even greater than his incentive to choose $p_{L}$ in $t=1$, when the fraction of type $H$ sellers in the market increases from $q_{0}$ to $q^{\prime}$. First, note by (18) that

$$
\pi_{b}^{L}\left(q^{\prime}, v_{L}^{0}, v_{b}^{0}\left[q^{+}\left(q^{\prime}, v_{L}^{0}\right)\right]\right)=\delta_{b} v_{b}^{0}\left(q^{\prime}\right)+\left(1-q^{\prime}\right) F\left(\frac{p_{L}}{v_{L}^{0}}\right)\left[u_{L}-p_{L}-\delta_{b}\left(u_{L}-p_{H}\right)\right] .
$$

Second, since $v_{b}^{1}\left(q^{\prime}\right) \geq v_{b}^{0}\left(q^{\prime}\right)=v_{b}^{0}\left(q^{+}\left(q_{0}, v_{L}^{1}\right)\right)$ by $(19)$, we have

$$
\begin{aligned}
\pi_{b}^{L}\left(q_{0}, v_{L}^{1}, v_{b}^{1}\left(q^{\prime}\right)\right) & \geq \pi_{b}^{L}\left(q_{0}, v_{L}^{1}, v_{b}^{0}\left(q^{\prime}\right)\right) \\
& =\delta_{b} v_{b}^{0}\left(q_{0}\right)+\left(1-q_{0}\right) F\left(\frac{p_{L}}{v_{L}^{1}}\right)\left[u_{L}-p_{L}-\delta_{b}\left(u_{L}-p_{H}\right)\right]
\end{aligned}
$$

the second equality follows from (18). Therefore,

$$
\begin{aligned}
& \pi_{b}^{L}\left(q^{\prime}, v_{L}^{0}, v_{b}^{0}\left[q^{+}\left(q^{\prime}, v_{L}^{0}\right)\right]\right)-\pi_{b}^{L}\left(q_{0}, v_{L}^{1}, v_{b}^{1}\left(q^{\prime}\right)\right) \\
& \quad \leq \delta_{b}\left[v_{b}^{0}\left(q^{\prime}\right)-v_{b}^{0}\left(q_{0}\right)\right]+\left\{\left(1-q^{\prime}\right) F\left(\frac{p_{L}}{v_{L}^{0}}\right)-\left(1-q_{0}\right) F\left(\frac{p_{L}}{v_{L}^{1}}\right)\right\}\left[u_{L}-p_{L}-\delta_{b}\left(u_{L}-p_{H}\right)\right] .
\end{aligned}
$$

Since $v_{L}^{0}>v_{L}^{1}, u_{L}<p_{H}$, and $q^{\prime}>q_{0}$, the second term on the right-hand side of the above inequality is negative, so that

$$
\pi_{b}^{L}\left(q^{\prime}, v_{L}^{0}, v_{b}^{0}\left[q^{+}\left(q^{\prime}, v_{L}^{0}\right)\right]\right)-\pi_{b}^{L}\left(q_{0}, v_{L}^{1}, v_{b}^{1}\left(q^{\prime}\right)\right)<\pi_{b}^{H}\left(q^{\prime}\right)-\pi_{b}^{H}\left(q_{0}\right)
$$


which confirms (20).

By (17), $q^{+}\left(q_{0}, v_{L}^{1}\right)$ is strictly increasing in $q$. Thus, (15) and (16) are satisfied if, and only if, $q_{0} \in\left[\underline{q}^{2}, \bar{q}^{2}\right]$, where

$$
q^{+}\left(\underline{q}_{2}, v_{L}^{1}\right)=\underline{q}_{1} \quad \text { and } \quad q^{+}\left(\bar{q}_{2}, v_{L}^{1}\right)=\bar{q}^{1}
$$

To finish the characterization of the 2 -step equilibria, we show that $\bar{q}^{2} \geq \underline{q}_{1}$. Suppose not and let $q^{\prime} \in\left(\bar{q}^{2}, \underline{q}^{1}\right)$. We know from Proposition 1 that there is an equilibrium $\sigma^{*}=\left\{\sigma_{t}^{*}\right\}_{t=0}^{\infty}$ when $q_{0}=q^{\prime}$. Moreover, by Proposition 2, the market clears in finite time in this equilibrium. Let $T$ be the period when the market clears and denote by $q_{t}$ the fraction of type $H$ sellers in the market in $t \in\{0, \ldots, T\}$. We know that $\left\{q_{t}\right\}_{t=0}^{T}$ is strictly increasing and, by Proposition 3 , that $q_{T} \geq \underline{q}^{0}>\underline{q}^{1}$. Hence, there is $T^{\prime} \in\{1, \ldots, T-1\}$ such that $q_{T^{\prime}-1}<\underline{q}^{1}$ and $q_{T^{\prime}} \geq \underline{q}^{1}$. Consider then the strategy profile $\hat{\sigma}^{*}=\left\{\sigma_{t}^{*}\right\}_{t=T^{\prime}-1}^{\infty}$. By construction, $\hat{\sigma}^{*}$ is a 2-step equilibrium when $q_{0}=q_{T^{\prime}-1}$, as $\bar{q}^{2}<q_{1}$ by assumption, and so $\hat{\sigma}^{*+}=\left\{\sigma_{t}^{*}\right\}_{t=T^{\prime}}^{\infty}$ must be a 1-step equilibrium with $q_{0}=q_{T^{\prime}}$. This, however, implies that $\bar{q}^{2} \geq q^{\prime}$, a contradiction.

We can now state and prove a complete characterization of the $k$-step equilibria for all $k \geq 2$. For this, define the sequences $\left\{v_{b}^{k}\right\}_{k=2}^{\infty},\left\{v_{L}^{k}\right\}_{k=2}^{\infty},\left\{\underline{q}^{k}\right\}_{k=2}^{\infty}$, and $\left\{\bar{q}^{k}\right\}_{k=2}^{\infty}$ recursively as follows:

$$
\begin{aligned}
& \text { (i) } v_{b}^{k}(q)=\pi_{b}^{L}\left(q, v_{L}^{k-1}, v_{b}^{k-1}\left[q^{+}\left(q, v_{L}^{k-1}\right)\right]\right) ; \\
& \text { (ii) } v_{L}^{k}=\int \max \left\{p_{L}, \delta_{s} v_{L}^{k-1}\right\} d F\left(\delta_{s}\right) \\
& \text { (iii) } \underline{q}^{k-1}=q^{+}\left(\underline{q}^{k}, v_{L}^{k-1}\right) \\
& \text { (iv) } \bar{q}^{k-1}=q^{+}\left(\bar{q}^{k}, v_{L}^{k-1}\right) .
\end{aligned}
$$

Notice that $\left\{v_{L}^{k}\right\}$ is non-decreasing and that $v_{L}^{k}<v_{L}^{k-1}$ if, and only if, $p_{L} / v_{L}^{k-1}<\bar{\delta}_{s}$. Consequently, if there is $k \geq 2$ such that $p_{L} / v_{L}^{k-1}<\bar{\delta}_{s}$, then $v_{L}^{k}<v_{L}^{k-1}<\cdots<v_{L}^{2}<v_{L}^{1}$. Also notice that $\underline{q}^{k-1}>\underline{q}^{k}$ and $\bar{q}^{k-1}>\bar{q}^{k}$ if, and only if $p_{L} / v_{L}^{k-1}<\bar{\delta}_{s}$.

Proposition 5. Fix $K \geq 2$ and suppose that $p_{L} / v_{L}^{K-1}<\bar{\delta}_{s}$. For each $k \in\{2, \ldots, K\}$ there exists a $k$-step equilibrium if, and only if, $q_{0} \in\left[\underline{q}^{k}, \bar{q}^{k}\right]$. Moreover, $\underline{q}^{k}<\underline{q}^{k-1} \leq \bar{q}^{k}<\bar{q}^{k-1}$ for all $k \in\{2, \ldots, K\}$. 
Proof. The proof is by induction on $K$. We know from above that the result is true when $K=2$ and that

$$
v_{b}^{2}\left(q_{0}\right)-\pi_{b}^{H}\left(q_{0}\right) \geq v_{b}^{1}\left[q^{+}\left(q_{0}, v_{L}^{1}\right)\right]-\pi_{b}^{H}\left(q^{+}\left(q_{0}, v_{L}^{1}\right)\right)
$$

Suppose then, by induction, that the result is true for some $K \geq 2$ and that

$$
v_{b}^{K}\left(q_{0}\right)-\pi_{b}^{H}\left(q_{0}\right) \geq v_{b}^{K-1}\left[q^{+}\left(q_{0}, v_{L}^{K-1}\right)\right]-\pi_{b}^{H}\left(q^{+}\left(q_{0}, v_{L}^{K-1}\right)\right)
$$

Moreover, assume that $p_{L} / v_{L}^{K}<\bar{\delta}_{s}$. Consider now a strategy profile $\sigma^{K+1}$ such that the buyers offer $p_{L}$ in the first $K+1$ periods, and then they offer $p_{H}$. By the induction hypothesis, the following conditions are necessary and sufficient for $\sigma^{K+1}$ to be an equilibrium:

$$
\begin{gathered}
q^{+}\left(q_{0}, v_{L}^{K}\right) \in\left[\underline{q}^{K}, \bar{q}^{K}\right] \\
\pi_{b}^{H}\left(q_{0}\right) \leq \pi_{b}^{L}\left(q_{0}, v_{L}^{K}, v_{b}^{K}\left[q^{+}\left(q_{0}, v_{L}^{K}\right)\right]\right) .
\end{gathered}
$$

By (23) and (24), condition (26) is satisfied if, and only if, $q_{0} \in\left[\underline{q}^{K+1}, \bar{q}^{K+1}\right]$. Also notice that $p_{L} / v_{L}^{K}<\bar{\delta}_{s}$ implies that $\underline{q}^{K+1}<\underline{q}^{K}$ and $\bar{q}^{K+1}<\bar{q}^{K}$. We are done if we show that: $(a)$ (26) implies (27); and $(b) \underline{q}^{K} \leq \bar{q}^{K+1}$. Item (a) follows from (25) and the induction hypothesis; the details are in the Appendix. Item $(b)$ follows from exactly the same argument used to prove that $\underline{q}^{1} \leq \bar{q}^{2}$.

\section{Information Frictions and Liquidity}

Given the cutoff $\underline{q}^{0}$ and the sequence of cutoffs $\left\{\underline{q}^{k}, \bar{q}^{k}\right\}$ for $k \geq 1$, with $\underline{q}^{k}<\underline{q}^{k-1} \leq \bar{q}^{k}$, we have provided a complete characterization of all equilibria for any $q_{0} \in(0,1)$. Though $\bar{q}^{1}>\underline{q}^{0}>\underline{q}^{1}$ implies the existence of multiple equilibria for some values of $q_{0}$, it also implies a natural monotonicity: for any $q_{0} \in(0,1)$, if there exists an equilibrium in which the market clears at time $T$, then for any $q_{0}^{\prime}>q_{0}$ there exists an equilibrium in which the market clears at some time $T^{\prime} \leq T$. In order to study the relationship between the severity of the information frictions (i.e. the fraction of low quality sellers, $1-q_{0}$ ) and market liquidity (i.e. 
the amount of time it takes for a market to clear, $T$ ) we focus on the equilibria that take the smallest number of periods before the market clears. ${ }^{15}$ Below we illustrate this relationship.

Figure 1: Information and Market Liquidity

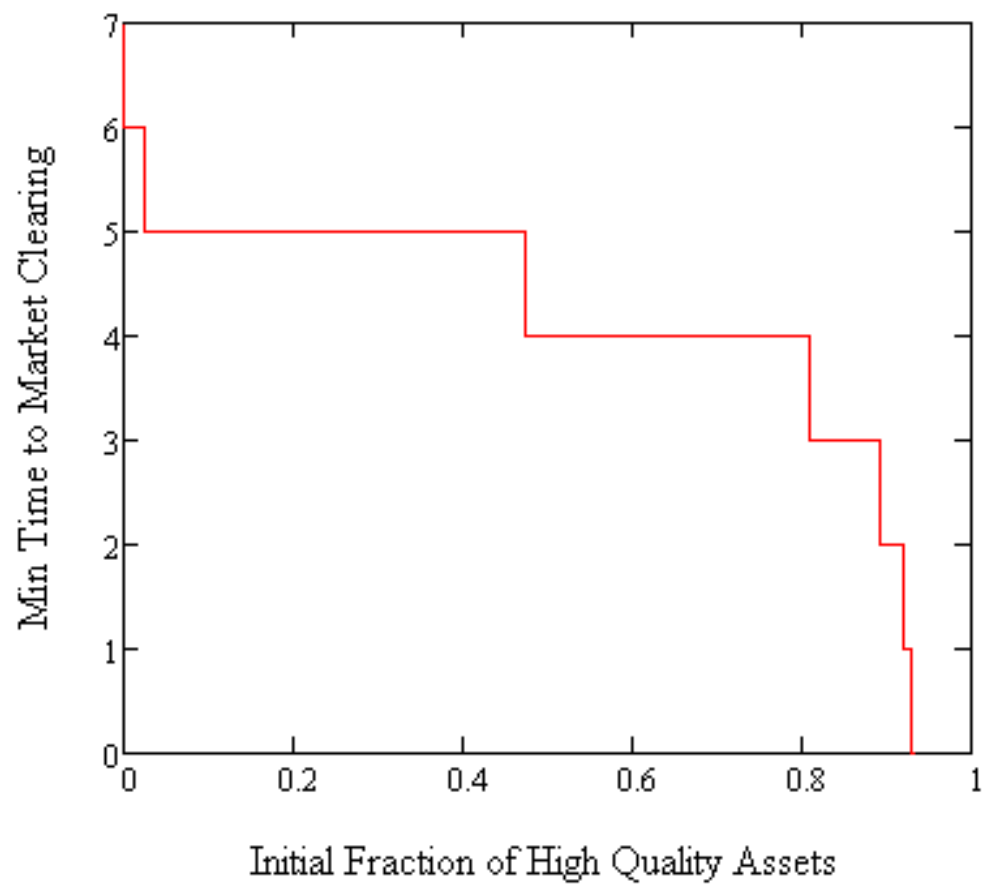

\footnotetext{
${ }^{15}$ This particular selection device is not essential for our comparative statics; given the monotonicity of equilibria, so long as we are consistent with the equilibria we select, our results will remain unchanged. However, this seems the most natural.
} 


\section{Appendix}

\subsection{Omitted Details from the Proof of Proposition 3}

From the argument in the main text, the only thing left to prove is that

$$
V_{t}^{b}\left(\widetilde{p}_{H} \mid \hat{\sigma}\right) \geq v_{b}^{0}(q)
$$

for all $t \geq 0$. For this, notice that

$$
V_{t}^{b}\left(\widetilde{p}_{H} \mid \hat{\sigma}, \alpha\right)=\sum_{\tau=1}^{\infty} \alpha(1-\alpha)^{\tau-1} \delta_{b}^{\tau-t} v_{b}^{0}\left(q_{t+\tau-1}^{\alpha}\right),
$$

where $q_{t+\tau-1}^{\alpha}$ is the fraction of type $H$ sellers in the market in period $t+\tau-1$ when the probability that an agent can trade in a period is $\alpha \in(0,1)$. Now observe that

$$
\begin{aligned}
& q_{t+1}^{\alpha}= \\
& \frac{q_{t}^{\alpha}\left\{1-\alpha+\alpha\left[1-F\left(\frac{p_{L}-y_{s}^{H}}{V_{t+1}^{H}(\hat{\sigma} \mid \alpha)}\right)\right]\right\}}{q_{t}\left\{1-\alpha+\alpha\left[1-F\left(\frac{p_{L}-y_{s}^{H}}{V_{t+1}^{H}(\hat{\sigma} \mid \alpha)}\right)\right]\right\}+\left(1-q_{t}\right)\left\{1-\alpha+\alpha\left[1-F\left(\frac{p_{L}}{V_{t+1}^{L}(\hat{\sigma} \mid \alpha)}\right)\right]\right\}} .
\end{aligned}
$$

Since $V_{t+1}^{H}(\hat{\sigma} \mid \alpha)>V_{t+1}^{L}(\hat{\sigma} \mid \alpha)$ if $\hat{\sigma}$ is to be an equilibrium (for a type $H$ seller can immitate a type $L$ seller and $\left.y_{s}^{H}>y_{s}^{L}\right)$, the sequence $\left\{q_{t}^{\alpha}\right\}_{t=0}^{\infty}$ is strictly increasing. Hence,

$$
V_{t}^{b}\left(\widetilde{p}_{H} \mid \hat{\sigma}, \alpha\right) \geq \sum_{\tau=1}^{\infty} \alpha(1-\alpha)^{\tau-1} \delta_{b}^{\tau-t} v_{b}^{0}(q),
$$

which implies the desired result.

\subsection{Omitted Details from the Proof of Proposition 5}

We need to prove that $q^{+}\left(q_{0}, v_{L}^{K}\right) \in\left[\underline{q}^{K}, \bar{q}^{K}\right]$ implies that

$$
\pi_{b}^{H}\left(q_{0}\right) \leq \pi_{b}^{L}\left(q_{0}, v_{L}^{K}, v_{b}^{K}\left[q^{+}\left(q_{0}, v_{L}^{K}\right)\right]\right) .
$$


For this, let $q^{\prime}=q^{+}\left(q_{0}, v_{L}^{K}\right)$ and $q^{\prime \prime}=q^{+}\left(q^{\prime}, v_{L}^{K}\right)$. First note that

$$
\begin{aligned}
& \pi_{b}^{L}\left(q_{0}, v_{L}^{K}, v_{b}^{K}\left(q^{\prime}\right)\right) \\
&=\left(1-q_{0}\right) F\left(\frac{p_{L}}{v_{L}^{K}}\right)\left[u_{L}-p_{L}\right]+\delta_{b}\left\{q_{0}+\left(1-q_{0}\right)\left[1-F\left(\frac{p_{L}}{v_{L}^{K}}\right)\right]\right\} v_{b}^{K}\left(q^{\prime}\right) \\
&=\left(1-q_{0}\right) F\left(\frac{p_{L}}{v_{L}^{K}}\right)\left[u_{L}-p_{L}\right]+\delta_{b}\left\{q_{0}+\left(1-q_{0}\right)\left[1-F\left(\frac{p_{L}}{v_{L}^{K}}\right)\right]\right\} v_{b}^{0}\left(q^{\prime}\right) \\
& \quad \quad \delta_{b}\left\{q_{0}+\left(1-q_{0}\right)\left[1-F\left(\frac{p_{L}}{v_{L}^{K}}\right)\right]\right\}\left[v_{b}^{K}\left(q^{\prime}\right)-v_{b}^{0}\left(q^{\prime}\right)\right] \\
&=\delta_{b} v_{b}^{0}\left(q_{0}\right)+\left(1-q_{0}\right) F\left(\frac{p_{L}}{v_{L}^{K}}\right)\left[u_{L}-p_{L}-\delta_{b}\left(u_{L}-p_{H}\right)\right] \\
& \quad+\delta_{b}\left\{q_{0}+\left(1-q_{0}\right)\left[1-F\left(\frac{p_{L}}{v_{L}^{K}}\right)\right]\right\}\left[v_{b}^{K}\left(q^{\prime}\right)-v_{b}^{0}\left(q^{\prime}\right)\right]
\end{aligned}
$$

where the last equality follows from (18). Similarly, one can show that

$$
\begin{aligned}
& \pi_{b}^{L}\left(q^{\prime}, v_{L}^{K-1}, v_{b}^{K-1}\left(q^{\prime \prime}\right)\right) \\
& =\delta_{b} v_{b}^{0}\left(q^{\prime}\right)+\left(1-q^{\prime}\right) F\left(\frac{p_{L}}{v_{L}^{K-1}}\right)\left[u_{L}-p_{L}-\delta_{b}\left(u_{L}-p_{H}\right)\right] \\
& \quad+\delta_{b}\left\{q^{\prime}+\left(1-q^{\prime}\right)\left[1-F\left(\frac{p_{L}}{v_{L}^{K-1}}\right)\right]\right\}\left[v_{b}^{K-1}\left(q^{\prime \prime}\right)-v_{b}^{0}\left(q^{\prime \prime}\right)\right] .
\end{aligned}
$$

Now observe, by (25), that

$$
\begin{aligned}
\pi_{b}^{L}\left(q_{0}, v_{L}^{K}, v_{b}^{K}\left(q^{\prime}\right)\right) \geq & \delta_{b} v_{b}^{0}\left(q_{0}\right)+\left(1-q_{0}\right) F\left(\frac{p_{L}}{v_{L}^{K}}\right)\left[u_{L}-p_{L}-\delta_{b}\left(u_{L}-p_{H}\right)\right] \\
& +\delta_{b}\left\{q_{0}+\left(1-q_{0}\right)\left[1-F\left(\frac{p_{L}}{v_{L}^{K}}\right)\right]\right\}\left[v_{b}^{K-1}\left(q^{\prime \prime}\right)-v_{b}^{0}\left(q^{\prime}\right)\right] .
\end{aligned}
$$

Hence,

$$
\begin{aligned}
\pi_{b}^{L}\left(q^{\prime}, v_{L}^{K-1}, v_{b}^{K-1}\left(q^{\prime \prime}\right)\right)-\pi_{b}^{L}\left(q_{0}, v_{L}^{K}, v_{b}^{K}\left(q^{\prime}\right)\right) \leq \delta_{b}\left[v_{b}^{0}\left(q^{\prime}\right)-v_{b}^{0}\left(q_{0}\right)\right] & \\
+\left\{u_{L}-p_{L}-\delta_{b}\left(u_{L}-p_{H}-\left[v_{b}^{K-1}\left(q^{\prime \prime}\right)-v_{b}^{0}\left(q^{\prime \prime}\right)\right]\right)\right\} & \left\{\left(1-q^{\prime}\right) F\left(\frac{p_{L}}{v_{L}^{K-1}}\right)\right. \\
- & \left.-\left(1-q_{0}\right) F\left(\frac{p_{L}}{v_{L}^{K}}\right)\right\} .
\end{aligned}
$$

Now observe that $q^{\prime}>q_{0}, v_{L}^{K}<v_{L}^{K-1}$, and

$$
\left(1-q^{\prime}\right) F\left(\frac{p_{L}}{v_{L}^{K-1}}\right)-\left(1-q_{0}\right) F\left(\frac{p_{L}}{v_{L}^{K}}\right)<0 .
$$


In addition, $u_{L}<p_{H}$ and $u_{L}-p_{L} \geq \delta_{b}\left(u_{H}-p_{H}\right)>\delta_{b}\left[v_{b}^{K-1}\left(q^{\prime \prime}\right)-v_{b}^{0}\left(q^{\prime \prime}\right)\right]$. Therefore, (29) implies that

$$
\begin{aligned}
\pi_{b}^{L}\left(q^{\prime}, v_{L}^{K-1}, v_{b}^{K-1}\left(q^{\prime \prime}\right)\right)-\pi_{B}^{L}\left(q_{0}, v_{L}^{K}, v_{b}^{K}\left(q^{\prime}\right)\right) & <v_{b}^{0}\left(q^{\prime}\right)-v_{b}^{0}\left(q_{0}\right) \\
& =\pi_{b}^{H}\left(q^{\prime}\right)-\pi_{b}^{H}\left(q_{0}\right)
\end{aligned}
$$

Since $\pi_{b}^{L}\left(q^{\prime}, v_{L}^{K-1}, v_{b}^{K-1}\left(q^{\prime \prime}\right)\right) \geq \pi_{b}^{H}\left(q^{\prime}\right)$ by the induction hypothesis, we can then conclude that (28) is satisfied. 


\section{References}

G.A. Akerlof. The Market for "Lemons": Quality Uncertainty and the Market Mechanism. The Quarterly Journal of Economics, 84(3):488-500, 1970.

M.R. Blouin. Equilibrium in a decentralized market with adverse selection. Economic Theory, $22(2): 245-262,2003$.

M.R. Blouin and R. Serrano. A decentralized market with common values uncertainty: Non-steady states. Review of Economic Studies, pages 323-346, 2001.

R. Deneckere and M.Y. Liang. Bargaining with interdependent values. Econometrica, 74(5): 1309-1364, 2006.

D. Duffie, N. Garleanu, and L.H. Pedersen. Over-the-counter markets. Econometrica, pages 1815-1847, 2005.

D. Duffie, N. Gârleanu, and L.H. Pedersen. Valuation in over-the-counter markets. Review of Financial Studies, 20(6):1865, 2007.

R. Evans. Sequential bargaining with correlated values. The Review of Economic Studies, pages 499-510, 1989.

D. Fudenberg, D. Levine, and J. Tirole. Infinite-horizon models of bargaining with one-sided incomplete information. Game theoretic models of Bargaining, pages 73-98, 1985.

D. Gale. Bargaining and Competition Part I: Characterization. Econometrica, 54(4):785806, 1986a.

D. Gale. Bargaining and Competition Part II: Existence. Econometrica, 54(4):807-818, 1986b.

N. Gârleanu. Portfolio choice and pricing in illiquid markets. Journal of Economic Theory, 144(2):532-564, 2009. 
F. Gul, H. Sonnenschein, and R. Wilson. Foundations of dynamic monopoly and the coase conjecture. Journal of Economic Theory, 39(1):155-190, 1986.

Y. Higashi, K. Hyogo, and N. Takeoka. Subjective random discounting and intertemporal choice. Journal of Economic Theory, 144(3):1015-1053, 2009.

R. Inderst. Matching markets with adverse selection. Journal of Economic Theory, 121(2): 145-166, 2005.

M. Janssen and S. Roy. Dynamic Trading in a Durable Good Market with Asymmetric Information. International Economic Review, 43:257-282, 2002.

D. Moreno and J. Wooders. Prices, delay, and the dynamics of trade. Journal of Economic Theory, 104(2):304-339, 2002.

D. Moreno and J. Wooders. Decentralized trade mitigates the lemons problem. International Economic Review, forthcoming, 2009.

A. Rubinstein and A. Wolinsky. Equilibrium in a Market with Sequential Bargaining. Econometrica, 53(5):1133-1150, 1985.

M. Satterthwaite and A. Shneyerov. Dynamic Matching, Two-Sided Incomplete Information, and Participation Costs: Existence and Convergence to Perfect Competition. Econometrica, 75(1):155-200, 2007.

D. Vayanos and P.O. Weill. A Search-Based Theory of the On-the-Run Phenomenon. The Journal of Finance, 63(3):1361-1398, 2008.

D.R. Vincent. Bargaining with common values. Journal of Economic Theory, 48(1):47-62, 1989.

C. Wilson. The nature of equilibrium in markets with adverse selection. The Bell Journal of Economics, 11(1):108-130, 1980.

A. Wolinsky. Information revelation in a market with pairwise meetings. Econometrica, pages 1-23, 1990. 